Radial and Nonradial Pulsations as Probes of Stellar Physics

ASP Conference Series, Vol. 259, 2002

C. Aerts, T.R. Bedding, \& J. Christensen-Dalsgaard, eds.

\title{
Unified NRP-Modelling of Be Stars ${ }^{1}$
}

\author{
Th. Rivinius, D. Baade \\ European Southern Observatory, Garching bei München, Germany
}

S. Štefl

Astronomical Institute, Academy of Sciences, Ondřejov, Czech Republic

\author{
M. Maintz
}

Landessternwarte Königstuhl, Heidelberg, Germany

\begin{abstract}
Recently, the line profile variability (lpv) of two low- $v \sin i$ Be stars, $\mu$ Cen and $\omega(28)$ CMa was successfully modelled as nonradial pulsation ( $n r p$ ) of rapidly rotating stars seen pole-on. In this work, it is shown that the $l p v$ of low $v$ sin $i$ early-type Be stars in general closely resembles these two cases, and is therefore explainable by the same mechanism. The $l p v$ of intermediate to high- $v \sin i$ Be stars can be explained by the same model if the inclination angle of the model alone is increased. Consequently, early-type Be stars form a distinct, fairly homogeneous class of non-radial low-order $g$-mode pulsators.
\end{abstract}

\section{Introduction}

In the past few years, several early-type Be stars have been monitored by us on timescales from hours to years, using high resolution echelle spectroscopy. Most of these stars show the signature of periodic line profile variability on timescales between 0.5 and 2 day, while in rapidly rotating non-Be stars such $l p v$ has not yet been seen.

The two most thoroughly investigated stars in the sample are $\mu$ Cen (Rivinius et al., 2001 and references therein) and $\omega \mathrm{CMa}$ (Maintz et al., 2000). $\mu$ Cen is a multiperiodic B2 IVe star, for which the $l p v$ could be modelled succesfully as $n r p$, using Townsend's (1997) BRUCE/KYLIE package. The apparently singlyperiodic lp $v$ of $\omega$ CMa was reproduced as nrp in unprecedented detail by Maintz et al. (2000 and Fig. 1), using the same model code. Both stars were found to pulsate in low-order $g$-modes with $\ell=m=+2$ (the four main modes in $\mu$ Cen).

Although the models of these two stars are surprisingly similar, it remained to be shown that they are representative of early-type Be stars. In order to do so, the lpv of $\omega \mathrm{CMa}$ is compared to that of other Be stars. Based on the

\footnotetext{
${ }^{1}$ Based on observations obtained at the European Southern Observatory, La Silla, Chile, prop. No. 64.H-0548, and Calar Alto observatory (DSAZ)
} 


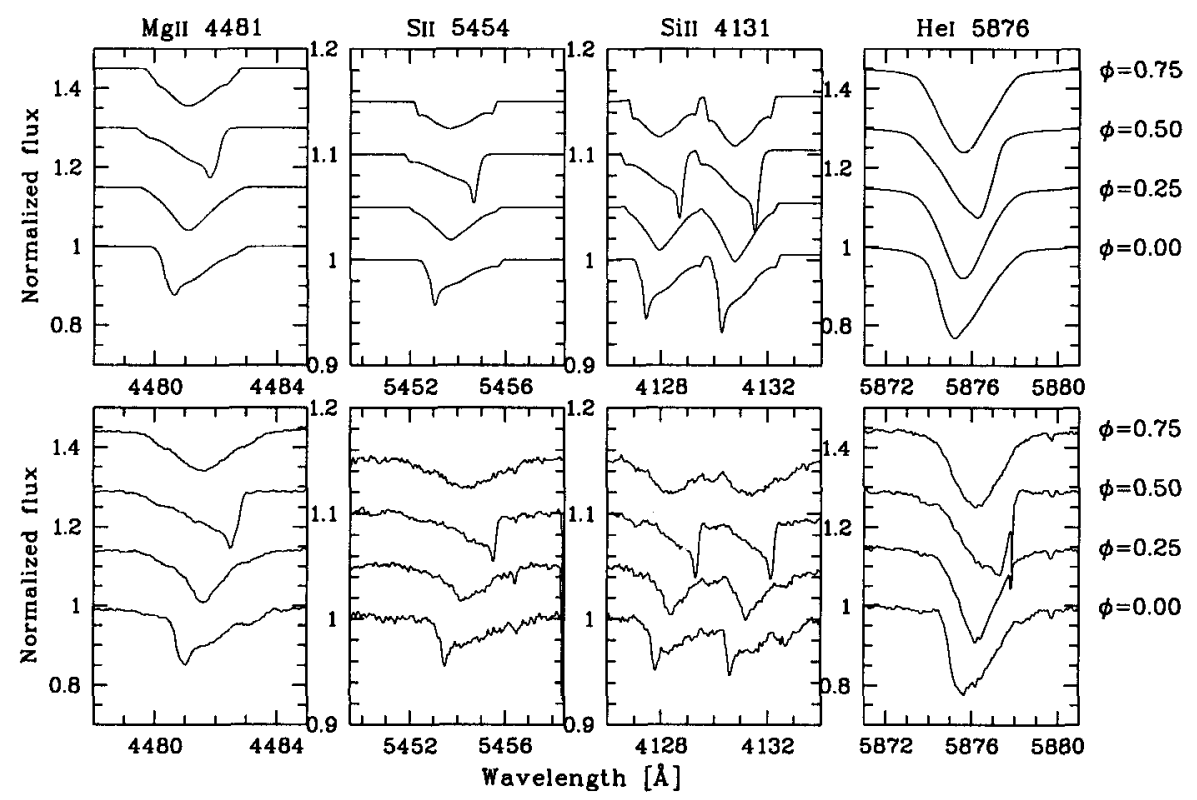

Figure 1. Modelled (top) and observed (bottom) $l p v$ for different ions in $\omega \mathrm{CMa}$, using the model parameters by Maintz et al. (2000).

assumption that Be stars are intrinsically rapid rotators, so that differences in $v \sin i$ are mainly due to inclination, it is shown that Be stars with the same spectral type and the same $v \sin i$ exhibit the same variability. In addition, the $l p v$ of Be stars with the same spectral type, but different $v \sin i$ are also reproduced very well by the model of $\omega \mathrm{CMa}$ if the inclination angle alone is adjusted.

\section{Comparing observations vs. models}

Due to $\mu$ Cen's potentially confusing multiperiodicity, we restrict ourselves to $\omega \mathrm{CMa}$. The results for $\mu$ Cen are similar, but need more space to be explained.

The lpv of $\omega \mathrm{CMa}$ is the strongest observed in any Be star. The three most outstanding properties are so-called "spikes" (prominently seen at phases 0.0 and 0.5 in Fig. 1), the extended "ramps" apparent at the same phases on the opposite sides of the profile, and the "backward travelling bumps" (Fig. 2). Especially in metal lines like MgII 4481, such features can easily be identified. Rivinius et al. (2001) give a more thorough introduction to these $l p v$ properties and provide an overview of the modelling technique used. The model parameters for $\omega \mathrm{CMa}$ were taken from Maintz et al. (2000).

To show that low $v \sin i$ Be stars in general resemble $\omega \mathrm{CMa}(v \sin i=$ $\left.80 \mathrm{~km} \mathrm{~s}^{-1}\right)$ in their $l p v, \mathrm{FWCMa}\left(v \sin i=40 \mathrm{~km} \mathrm{~s}^{-1}\right)$ and HR $4625(v \sin i=$ 

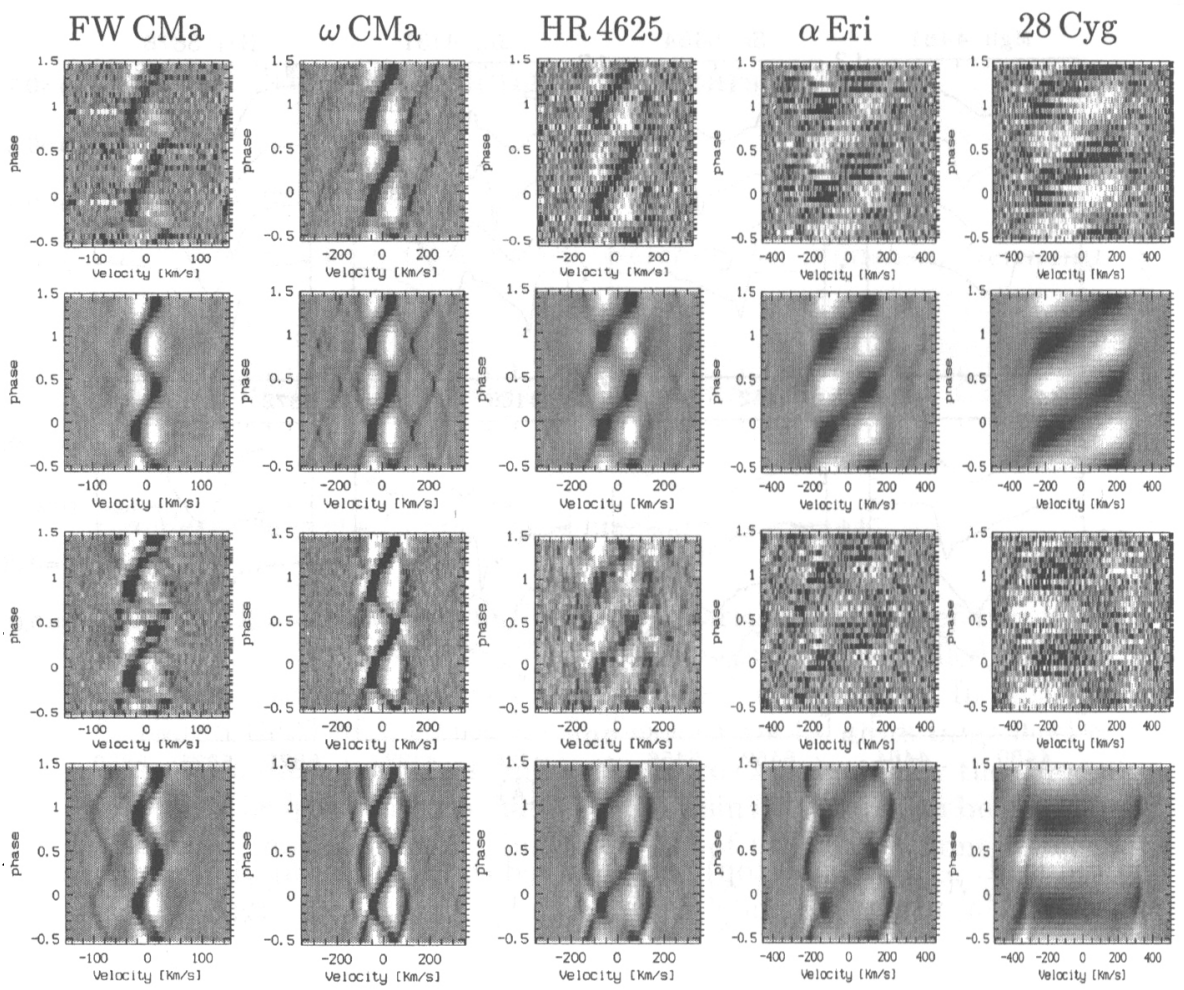

Figure 2. lpv observed in five different stars compared to the model of $\omega \mathrm{CMa}$ tilted to different inclination angles (Table 1). Top row: observed HeI 4388, second row: modelled HeI 4388, third row: observed MgII 4481, bottom row: modelled MgII 4481. Note that the apparently asymmetric lpv of MgII 4481 in $28 \mathrm{Cyg}$ is caused by rotational blending with HeI 4471.

$130 \mathrm{~km} \mathrm{~s}^{-1}$ ) were chosen, for which our archives hold most data. However, observations of other stars like HR $4074\left(v \sin i=80 \mathrm{~km} \mathrm{~s}^{-1}\right.$, see Štefl et al. these proceedings), HR $5223\left(v \sin i=70 \mathrm{~km} \mathrm{~s}^{-1}\right)$, or $31 \mathrm{Peg}\left(v \sin i=100 \mathrm{~km} \mathrm{~s}^{-1}\right)$ could also have been selected and will be presented in future publications.

As Fig. 2 shows, the variability in HeI 4388 is very similar for all three stars. To match the $v \sin i$ of the other two stars, the model for $\omega \mathrm{CMa}$ (Maintz et al., 2000) was tilted to other inclinations.

Differences between $\omega \mathrm{CMa}$ and these other stars, e.g., the lower prominence of the backward travelling bump in HR 4625, are well reproduced. Also the fact that features that are prominent in the high-amplitude star $\omega \mathrm{CMa}$ are generally weaker in FW CMa and HR 4625, agrees well with the strength of spikes and ramps being very sensitive to the pulsation amplitude (Maintz et al., 2000 and Rivinius et al., 2001). 
Table 1. Spectral types, periods and rotational velocities of the five example stars. The last column lists the inclination, at which the model for $\omega \mathrm{CMa}$ has to be viewed, to obtain the same $v \sin i$ as the object

\begin{tabular}{lcccc}
\hline $\begin{array}{l}\text { Star } \\
\text { name }\end{array}$ & $\begin{array}{c}\text { Spec. } \\
\text { type }\end{array}$ & $\begin{array}{c}\text { Period } \\
{[\mathrm{d}]}\end{array}$ & $\begin{array}{c}v \sin i \\
{\left[\mathrm{~km} \mathrm{~s}^{-1}\right]}\end{array}$ & $\begin{array}{c}\text { model } \\
\text { incl. }\end{array}$ \\
\hline FW CMa & B 3 Ve & 0.84 & 40 & $6.5^{\circ}$ \\
$\omega \mathrm{CMa}$ & B 2 IV-Ve & 1.37 & 90 & $15^{\circ}$ \\
HR 4625 & B 3 IVe & 1.68 & 130 & $22^{\circ}$ \\
$\alpha$ Eri & B 3 IVe & 1.28 & 220 & $39^{\circ}$ \\
$28 \mathrm{Cyg}$ & B 3 IVe & 0.65 & 320 & $66^{\circ}$ \\
\hline
\end{tabular}

Such distinct features disappear in high- $v \sin i$ Be stars as $\alpha$ Eri $(v \sin i=$ $\left.220 \mathrm{~km} \mathrm{~s}^{-1}\right)$ and $28 \mathrm{Cyg}\left(v \sin i=320 \mathrm{~km} \mathrm{~s}^{-1}\right)$. Again, only two objects out of several are shown for brevity. The backward-moving bumps disappear entirely and the ramps, although marginally present in the models, are below the $S / N$ of the observations. Both observed and modelled lpv signatures become weaker as $v \sin i$ increases despite the fact that in this comparison the amplitude of the model was not changed.

\section{Conclusions}

The lpv of the low- $v \sin i \omega \mathrm{CMa}$, which was modelled sucessfully as nrp by Maintz et al. (2000), could be shown to be representative of early-type Be stars in general. Other low- $v \sin i$ objects exhibit the same type of variability. The few differences are explainable by model inclination and amplitude. But otherwise the same parameters as for $\omega \mathrm{CMa}$ are appropriate. In high- $v \sin i$ Be stars the observed variability differs from that of $\omega \mathrm{CMa}$. But if the $n r p$ model for $\omega \mathrm{CMa}$ is viewed at higher inclinations, it again approximates well these observations.

The comparison of the observations of various low- $v \sin i$ Be stars supports the representative character of $\omega \mathrm{CMa}$, independently of the underlying mechanism. The comparison of the nrp model to high- $v \sin i$ Be stars demonstrates that $n r p$ is the explanation of the periodic $l p v$ of all early-type Be stars. They form a distinct and highly homogeneous group of low-order $g$-mode pulsators.

\section{References}

Maintz, M., Rivinius, Th., Tubbesing, S., Wolf, B., Štefl, S., \& Baade, D. 2000, in ASP Conf. Ser., Vol. 214, The Be Phenomenon in Early-Type Stars, eds. M. Smith, H. Henrichs, \& J. Fabregat, (San Francisco ASP), 244

Rivinius, Th., Baade, D., Štefl, S., Townsend, R. H. D., Stahl, O., Wolf, B., \& Kaufer, A. 2001, A\&A, 369, 1058

Townsend, R.H.D. 1997, MNRAS, 284, 839 